BNL 51302

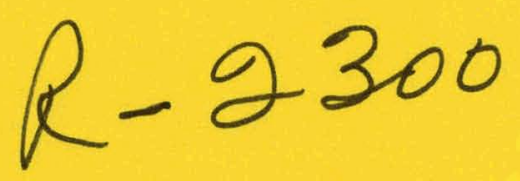

\title{
LONGITUDINAL SYMMETRIC COUPLED BUNCH MODES
}

\author{
J.M. WANG
}

December 1980

\section{ACCELERATOR DEPARTMENT}

BROOKHAVEN NATIONAL LABORATORY

ASSOCIATED UNIVERSITIES, INC.

UPTON, NEW YORK 11973

UNDER CONTRACT NO. DE-AC02-76CH00016 WITH THE

UNITED STATES DEPARTMENT OF ENERGY 


\section{DISCLAIMER}

This report was prepared as an account of work sponsored by an agency of the United States Government. Neither the United States Government nor any agency Thereof, nor any of their employees, makes any warranty, express or implied, or assumes any legal liability or responsibility for the accuracy, completeness, or usefulness of any information, apparatus, product, or process disclosed, or represents that its use would not infringe privately owned rights. Reference herein to any specific commercial product, process, or service by trade name, trademark, manufacturer, or otherwise does not necessarily constitute or imply its endorsement, recommendation, or favoring by the United States Government or any agency thereof. The views and opinions of authors expressed herein do not necessarily state or reflect those of the United States Government or any agency thereof. 


\section{DISCLAIMER}

Portions of this document may be illegible in electronic image products. Images are produced from the best available original document. 


\title{
MASTER
}

\section{LONGITUDINAL SYMMETRIC COUPLED BUNCH MODES}

\author{
J.M. WANG
}

December 1980

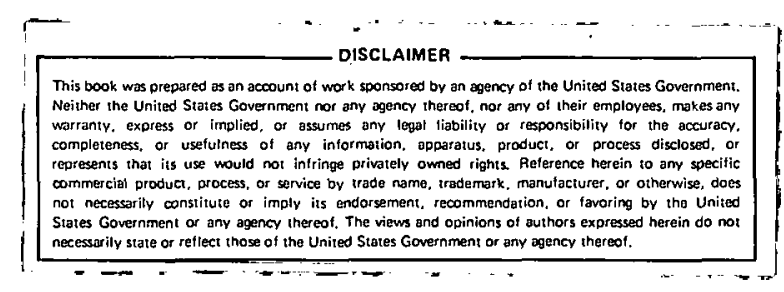

\section{ACCELERATOR DEPARTMENT}

rai

BROOKHAVEN NATIONAL LABORATORY UPTON, NEW YORK 11973 


\section{DISCLAIMER}

'I'his report was prepared as an account of work sponsored by an agency of the United Stales Government. Neither the United Siates Finvernment nor any agency thereif: nor any of their employees, nor any of their contractors, subcontractors, or their employees, makes any warranty, express or implied, or assumes any legal liability or responsibility for the accuracy, completeness, or usefulness of any information, apparatus, product, or process disclosed, or represents that its use would not infringe privately owned rights. Reference herein to any specific rommercial product, process, or service by trade name, trademark, manufacturer, or otherwise, does not necessarily woustifite ur inply it endorsement, rccommendaliui, ur lavuring by the United States Government or any agency, contractor or subcontractor thereof. The views and opinions of authors expressed herein do not necessarily state or reflect those of the United States Government or any agency, contractor or subcontractor thereof.

Printed in the United States of America

$$
\text { Available, from }
$$

National Technical Information Service

U.S. Department of Commerce 5285 Port Roýal Roád Springfield, VA 22161

Price: Printed Copy $\$ 5.00$; Microfiche $\$ 3.50$ 


\section{ABSTRACT}

The theory of longitudinal coupled bunch instabilities is discussed. Although some of the results included are not new 1 , it is useful to summarize them here in a unified fashion. 


\section{VLASOV EQUATION}

Let $\psi^{\mu}(\phi, \dot{\phi}, t)$ be the distribution function of the $\mu-t h$ bunch, $\mu=1,2,3 \ldots h$, in phase space, where $\phi=$ azimuthal angle:- $\omega_{0} t$, and $\dot{\phi}=\mathrm{d} \phi / \mathrm{d} t$. The total distribution function $\psi(\phi, \dot{\phi}, \mathrm{t})$ is related to $\psi^{\mu}(\phi, \dot{\phi}, t)$ by

$$
\psi(\phi, \dot{\phi}, t)=\sum_{\mu=1}^{h} \psi^{\mu}(\phi, \dot{\phi}, t)
$$

The Vlasov equation for the $\mu$-th bunch can be written as

$$
\frac{\partial \psi^{\mu}}{\partial t}+\frac{\partial \psi^{\mu}}{\partial \phi}-\omega_{S}^{2}\left(\phi-\phi_{O}^{\mu}\right) \frac{\partial \psi^{\mu}}{\partial \dot{\phi}}-\frac{n \omega_{O}}{\mathrm{p}} F(\phi, t) \frac{\partial \psi^{\mu}}{\partial \dot{\phi}}=0,
$$

where $\phi_{0}^{\mu} \equiv(2 \pi / h) \mu$ describes the position of the center of. the bunch, and $n$ is the frequency slip factor. The last term in Eq. (2) describes the interaction among the bunches. The function $F(\phi, t)$ will be given below.

Define the polar coordinates $\left(r_{\mu}, \theta_{\mu}\right)$ by the following:

$$
\begin{aligned}
\phi-\phi_{O}^{\mu} & =r_{\mu} \cos \theta_{\mu}, \\
\ddot{\phi} & =-\omega_{s} r_{\mu} \sin \theta_{\mu} .
\end{aligned}
$$

For symmetric coupled bunch modes, we can express the distribution function $\psi^{\mu}$ in terms of the polar variables as

$$
\psi^{\mu}(\phi, \dot{\phi}, t)=\psi_{0}\left(r_{\mu}\right)+\psi_{1}^{\mu}\left(r_{\mu}, \theta_{\mu}\right) e^{-i \Omega t}, \mu=1,2, \ldots h,
$$

where $\Omega$ is the coherent frequency. The unperturbed distribution function $\psi_{o}$ is the same in form for all $\mu$, since we are dealing with identical bunches.

If we ignore the term which describes the change in the equilibrium (time independent) distribution of lle lunches duc to their interaction with the environment, then, to the first order in F, Eq. (2) becomes

$$
-i \Omega \psi_{1}^{\mu}+\omega_{s} \frac{\partial \psi_{1}^{\mu}}{\partial \theta_{\mu}}+\frac{n \omega_{o}}{p} F\left(\phi_{o}^{\mu}+r_{\mu} \cos \theta_{\mu}\right) \frac{\sin \theta_{\mu}}{\omega_{s}} \psi_{o}\left(r_{\mu}\right)=0 .
$$

The function $F(\phi)$ is defined to be the force which the beam exerts on itself through the environment. It is proportional to the sum of the products of the Fourier components of the line density and the 
impedance at the corresponding frequencies. Thus, in our notation,

$$
\begin{aligned}
F(\phi) & =-\frac{\omega_{0}^{2}}{4 \pi^{2} B c} \int_{0}^{2 \pi} \mathrm{d} \phi^{\prime} \int_{-\infty}^{\infty} \mathrm{d} \phi^{\prime} \sum_{\nu=1}^{h} \psi_{1}^{\mu}\left(\phi^{\prime}, \phi^{\prime}\right) \sum_{n=-\infty}^{\infty} z_{n} e^{i n\left(\phi^{-} \phi^{\prime}\right)}, \\
& =-\frac{\omega_{0}^{2} \omega_{s}}{4 \pi^{2} B c} \sum \sum_{n} z_{n} \int_{0}^{2 \pi} r^{\prime} d^{\prime} \int_{0}^{2 \pi} d \theta^{\prime} \psi_{1}^{\nu}\left(r^{\prime}, \theta^{\prime}\right) e^{i n\left(\phi-r^{\prime} \cos \theta^{\prime}\right)}
\end{aligned}
$$

where we have used the abbreviation

$$
Z_{n} \equiv Z\left(n \omega_{0}+\Omega\right)
$$

$Z(\omega)$ being the impedance at frequency $\omega$.

\section{MULTIPOLE . EXPANSION}

We now examine the a-th multipole mode of coupled bunch oscillations:

$$
\psi_{1}^{\mu}\left(r_{\mu}, \theta_{\mu}\right)=\xi_{R}^{\mu}\left(r_{\mu}\right) e^{i a \theta_{\mu}},
$$

where $a$ is any integer, and $a= \pm 1$ corresponds to the dipole modes, $a= \pm 2$ to the quadrupole modes, etc. It can be shown that, if the frequency shift $\Delta \Omega=\Omega-a \omega_{s}$ is small compared to the synchrotron frequency $\omega_{S}$, the different multipole modes approximately decouple, and therefore it is justifiable to consider different multipole modes separately.

The form of the function $R$ in $\mathrm{Eq}$. (8) is independent of $\mu$, since we are considering symmetric coupled bunch modes. The relative phases of the bunches are described by the constants $\xi^{\mu},\left|\xi^{\mu}\right|$. being independent of $\mu$.

Substituting (8) into (7) and (5), we nbtain

$$
\begin{aligned}
\left(\Omega-a \omega_{s}\right) R(r) \xi^{\mu}= & -i a \frac{n \omega_{0}^{3}}{2 \pi p \beta c} \frac{\psi_{0}^{\prime}(r)}{r} \sum_{\nu, n} \xi^{\nu} \frac{z_{n}}{n} e^{i n\left(\phi_{0}^{\mu}-\phi_{0}^{\nu}\right)} \\
& \quad x J_{a}(n r) \int_{0}^{\infty} r^{\prime} d r^{\prime} R\left(r^{\prime}\right) J_{a}\left(n r^{\prime}\right) .
\end{aligned}
$$

In obtaining Eq. (9), we used the following identities:

$$
\frac{1}{2 \pi} \int_{0}^{2 \pi} d \theta^{\prime} e^{-i n r^{\prime} \cos \theta^{\prime}} e^{i a \theta^{\prime}}=(-i)^{a} J_{a}\left(n r^{\prime}\right)
$$

and

$$
\frac{1}{2 \pi} \int_{0}^{2 \pi} d \theta \sin \theta e^{i n r \cos \theta} e^{-i a \theta}=-a(-i)^{a} \frac{J_{a}(n r)}{n r} .
$$


Equation (9) defines the vector $\vec{\xi} \equiv\left(\xi^{1}, \xi^{2}, \ldots \xi^{\mathrm{h}}\right)$ to be an eigenvector of an $h \times h$ dimensional matrix. The matrix is a cyclic matrix. since $e^{i n}\left(\phi_{O}^{\mu}-\phi_{0}^{\nu}\right)=\exp [i 2 \pi n(\mu-\nu) / h]$. The solutions to such an equation are ${ }^{2}$

$$
\xi^{\mu}=e^{i} \frac{2 \pi}{h} s \mu,
$$

where the parameter $s, s=1,2, \ldots h$, characterizes different solutions. Substituting (10) into (9), and summing over $\nu$, one obtains:

$$
\begin{gathered}
\left(\Omega-a \omega_{s}\right) R(r)=-i a \frac{n \omega_{0}^{3 h}}{2 \pi p \beta c} \frac{\psi_{0}(r)}{r} \cdot \sum_{n=-\infty}^{\infty} \frac{Z_{n h+s}}{n h+s} J_{a}[(n h+s) r] \\
\quad \times \int_{0}^{\infty} r^{\prime} d r^{\prime} R\left(r^{\prime}\right) J_{a}\left[(n h+s) r^{-}\right] .
\end{gathered}
$$

Remember that the impedance $Z_{n}$ satisfies the following identity:

$$
z_{n}^{*}=z^{*}\left(n \omega_{0}+\Omega\right)=z\left(-n \omega_{0}-\Omega^{*}\right)
$$

Using Eq. (12) we observe that the solutions of Eq. (11) corresponding to the modes $(-a,-s)$ and $(a, s)$ are the complex conjugates of each other. We shall therefore consider only the modes with positive a.

There is little hope of solving (11) for $\Omega$ and $R(r)$ without some approximations. We adopt the small bunch approximation:

$$
J_{a}(n r) \simeq \frac{1}{2^{a} a !}(n r)^{a}
$$

Let us first use (13) for the Bessel function in (11) outside of the integral. The result is

$$
\begin{aligned}
\left(\Omega-a \omega_{s}\right) R(r)= & -i \frac{\mathrm{n} \omega^{3 h}}{2 \pi p \beta c} \frac{1}{2^{a}(a-1) !} r^{a-1} \psi_{0}^{\prime}(r) \sum_{h}(n h+s)^{a-1} z_{n h+s} \\
& \times \int_{0}^{\infty} r^{\prime} d r^{\prime} R\left(r^{\prime}\right) J_{a}\left[(n h+s) r^{-}\right]
\end{aligned}
$$

Equation (14) is the basis of the discussions in the following sections. 


\section{FREQUENCY SHIFT}

In this section, we assume that the synchrotron angular frequency $\omega_{s}$ is independent of the synchrotron amplitude $r$ :

$$
\omega_{s}(r)=\omega_{s o},
$$

where $\omega_{\text {so }}$ is the synchrotron frequency at vanishing amplitude. Under this assumption, the frequency shift $\Delta \Omega \equiv \Omega-$ a $\omega_{\text {so }}$ can easily be calculated.

By observation, the solution to $\mathrm{Eq} \cdot(14)$ is

$$
R(r)=\text { constant } \times r^{a-1} \psi_{0}^{\prime}(r)
$$

or equivalent1y,

$$
\psi^{\mu}\left(r_{\mu}, \theta_{\mu}\right)=\text { constant } \cdot \xi^{\mu} r_{\mu}^{a-1} \psi_{n}^{\prime}\left(r_{1 ! !}\right) e^{i a \theta} \mu .
$$

Substituting (15) into (14), we obtain

$\Delta \Omega=-i \frac{n \omega_{0}^{3} h}{2 \pi E} \frac{1}{2^{a}(a-1) ! n} \sum(n h+s)^{a-1} z_{n h+s} \int_{0}^{\infty} d r r^{a} \psi_{o}^{\prime}(r) J_{a}[(n h+s) r]$.

The above results can easily be understood. Equation (1.5 ) describes the situation where the a-th multiple pole component of the distorted. bunches is rotating rigldly in the phase space. The rotating distorted bunches excite electromagnetic field in the ring through the impedance $Z$, which in turn causes the frequency shift as given by Eq. (16). Note that the integral in (16) is nothing but the ( $\mathrm{nh}+\mathrm{s}$ )-th Fourier component of the line charge density corresponding to the phase space diotribution $\left(15^{\circ}\right)$.

Let us now specialize to the case of a Gaussian bunch:

$$
\psi_{0}(r)=\frac{N_{B}}{2 \pi \omega_{S O} L^{2}} e^{-r^{2} / 2 L^{2}}
$$

where $L$ is the rms half bunch length in units of radians, and $N_{B}$ is the number of particles per bunch, $\mathrm{N}_{\mathrm{B}} \equiv \mathrm{N} / \mathrm{h}$.

The integration in (16) ean le dune, giving

$$
\Delta \Omega=i \frac{n I_{0} \omega_{0}^{2}}{2 \pi E \omega_{\text {so }}} \frac{1}{2^{a}(a-1) !} L^{2 a-2} z_{\text {eff }}
$$


where

$$
\mathrm{Z}_{\text {eff }} \equiv \sum_{n=-\infty}^{\infty}(\mathrm{nh}+\mathrm{s})^{2 \mathrm{a}-1} \mathrm{z}\left[(\mathrm{nh}+\mathrm{s}) \omega_{\mathrm{o}}+\Omega\right] \mathrm{e}^{-\frac{1}{2}(\mathrm{nh}+\mathrm{s})^{2} \mathrm{~L}^{2}}
$$

and $I_{0} \equiv(1 / 2 \pi) N \omega_{0}$ is the total average current.

In the limit $\mathrm{L} \rightarrow 0$, Eq. (19) becomes

$$
\mathrm{Z}_{\text {eff }}=\sum_{\mathrm{n}=-\infty}^{\infty}(\mathrm{nh}+\mathrm{s})^{2 \mathrm{a}-1} \mathrm{Z}\left[(\mathrm{nh}+\mathrm{s}) \omega_{\mathrm{o}}+\Omega\right] .
$$

The approximation of (19) by (20) is equivalent to approximating the Bessel function in (16) by (13).

\section{LANDAU DAMPING}

When the synchrotron frequency $\omega_{s}$ is a function of the synchrotron amplitude $r$, the resulting frequency spread provides Landau damping.

To take into account the Landau damping, all we have to do is to regard $\omega_{\mathbf{s}}$ in $\mathrm{Eq}$. (14) as a function of $\mathrm{r}$; thus we have

$$
\begin{gathered}
R(r)=-i \frac{n \omega_{0}^{3} h}{2 \pi E} \frac{1}{2^{a}(a-1) !} \frac{r^{a} \psi_{o}^{-}(r)}{\Omega-a \omega_{s}(r)} \sum_{n}(n h+s)^{a-1} z_{n h+s} \\
\quad \times \int_{0}^{\infty} r^{-} d r^{\prime} R\left(r^{-}\right) J_{a}\left[(n h+s) r^{-}\right] .
\end{gathered}
$$

From observation of (21), one obtains immediately

$$
R(r)=\text { constant } \cdot r^{a-1} \psi_{o}^{-}(r) /\left[\Omega-a \omega_{s}(r)\right] .
$$

With use of Eq. (22), Eq. (21) becomes

$$
\begin{aligned}
1= & -i \frac{n \omega_{0}^{3} h}{2 \pi p \beta c} \frac{1}{2^{a}(a-1) ! n} \sum(n h+s)^{a=1} z_{n h+s} \\
& \times \int_{0}^{\infty} d r \frac{r^{a} \psi_{o}^{-}(r)}{\Omega-a \omega_{s}(r)} J_{a}[(n h+s) r] .
\end{aligned}
$$

Now approximate the Bessel function in (23) by using (13). The result is

$$
1=-i \frac{n \omega_{0}^{3} h}{2 \pi p \beta c} a\left[\frac{1}{2^{a} a !}\right]_{\text {eff }}^{2} \int_{0}^{\infty} \mathrm{dr} \frac{r^{2 a} \psi_{o}^{\prime}(r)}{\Omega-a \omega_{s}(r)} .
$$


Assume that all the non-linearity of the synchrotron frequency. is provided by the "natural" rf voltage,

$$
V(t)=\hat{V} \sin \left(h \omega_{0} t\right) \text {, }
$$

and that the bunch length is so small that it is sufficient to approximate $\mathrm{Eq}$. (25) by

$$
V(t)=\hat{V}\left[h \omega_{0} t-\frac{1}{6}\left(h \omega_{0} t\right)^{3}\right]
$$

then the amplitude dependence of the synchrotron frequency is given by ${ }^{3}$

$$
\omega_{S}(r)=w_{s o}\left(1-\frac{1}{16} r^{2}\right)
$$

For Gaussian bunches, we substitute Eqs. (17) and (26) into Eq. (24); we obtain as a result

$$
1=i \frac{n I_{o}}{\pi p \beta c}\left(\frac{\omega_{0}}{\omega_{s o}}\right)^{2} 2^{2-a}(a !)^{-2} L^{2 a-4} z_{e f f}[G(\Omega)]^{-1}
$$

where

$$
\left[G_{a}(\Omega)\right]^{-1} \fallingdotseq \int_{0}^{\infty} d x \frac{x^{a} e^{-x}}{x-y}
$$

and

$$
y \equiv \frac{8}{a \omega_{\text {so }} L^{2}}\left(a \omega_{s o}-\Omega\right)
$$

The threshold curve of the instability corresponds to the solution of Eq. (27) in the limit of $\mathrm{I}_{\mathrm{m}^{\prime}} \rightarrow 0^{+}$. In Figs. 1 and $2, \operatorname{Re}\left(G_{a}\right)$ is plotted against $\operatorname{Im}\left(\mathrm{G}_{a}\right)$ for the cases $a=1$ and $a=2$ respectively.

The threshold impedance, $\mathrm{Z}_{\mathrm{th}}^{\mathrm{th}}$, in the sense of Keil and Schnell corresponds to the minimum of $\left|G_{a}\right|$ along this curve. Thus

$$
F_{a}=\frac{n I}{\pi p \beta c}\left(\frac{\omega_{0}}{\omega_{s o}}\right)^{2} 2^{2-a}(a !)^{-2} L^{2 a-4}\left|z_{e f f}^{t h}\right|
$$

where

$$
\begin{aligned}
& F_{1}=0.78 \\
& F_{2}=0.56 .
\end{aligned}
$$




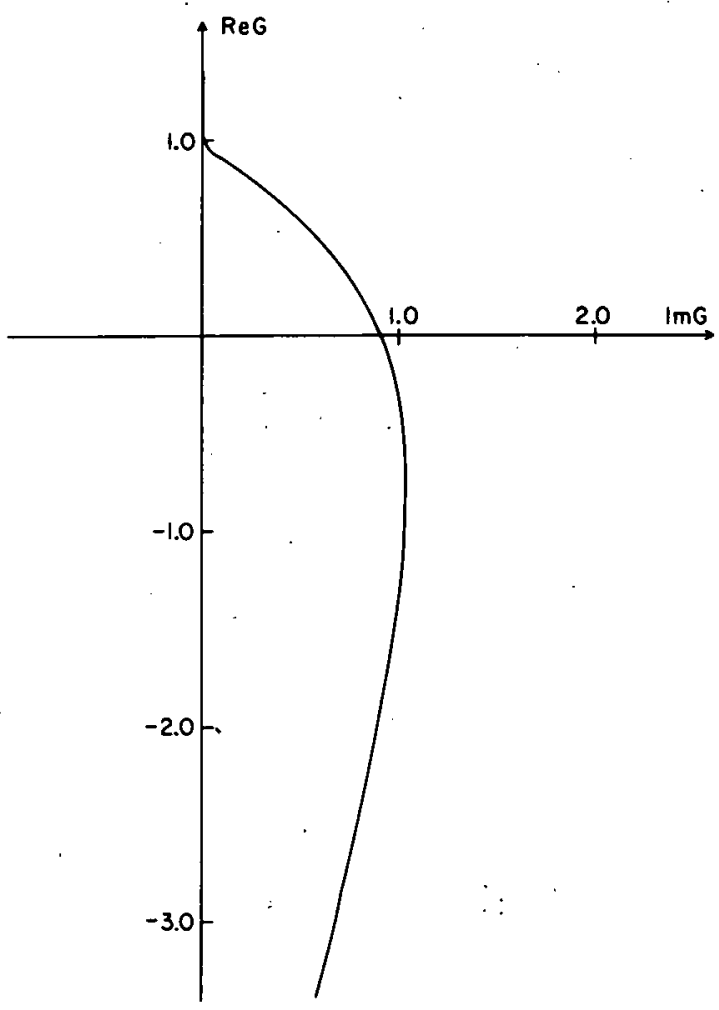

Fig. 1. Dipole mode. Stability boundary.

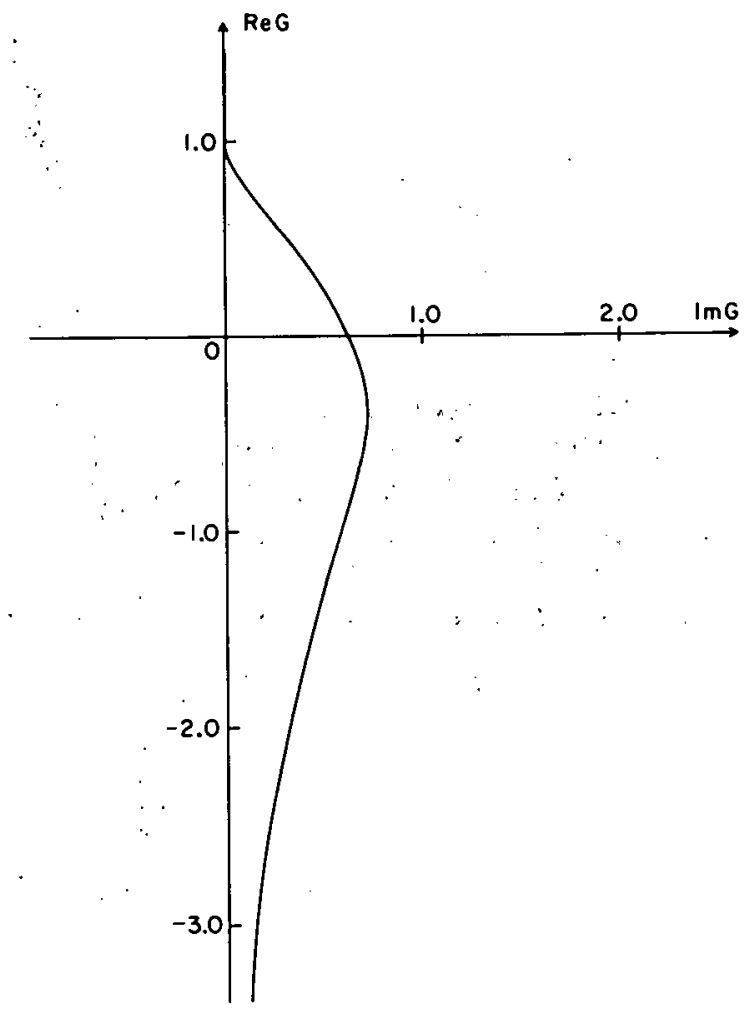

Fig. 2. Quadrupole mode. Stab1lity boundary. 
It often happens that the effective impedance $z_{\text {eff }}$ is predominantly inductive. Namely,

$$
\begin{gathered}
\operatorname{Re} Z_{\text {eff }}>0, \text { Im } z_{\text {eff }}<0 \text {, and } \\
\left|\frac{\operatorname{Re} Z_{\text {eff }}}{\operatorname{Im} Z_{\text {eff }}}\right|<1 .
\end{gathered}
$$

Such a phenomenon happens for the resistive wall contributions at ISABELLE. In a circumstance like this, we should take $F_{1}=F_{2}=1$. Let us now rewrite the stability condition

$$
\left|z_{\text {eff }}\right|<\left|z_{\text {eff }}^{\text {th }}\right|
$$

In terms of the frequency shift $\Delta \Omega$ as given by Eq. (18) and the synchrotron frequency spread $\delta \omega_{s}$ defined as

$$
\delta \omega_{s}=\frac{1}{16} \omega_{s o} \cdot(2.5 L)^{2} \text {. }
$$

Compare (32) and (26); we are defining the "total" frequency spread. $\delta \omega_{s}$ as the $2.5 \sigma$ value.

The stability criterion (31) becomes

$$
\frac{|\Delta \Omega|}{\delta \omega_{1}}<0.32 \mathrm{a}(\mathrm{a} !) \mathrm{F}_{\mathrm{a}}
$$

\section{ACKNOWLEDGEMENT}

The author thanks C. Pellegrini for discussions.

\section{REFERENCES}

1. M.Q. Barton and E.C. Raka, IEEE Trans. Nuclear Sci. NS-18, 1032

(1971); F. Sacherer, IEEE Trans. Nuclear Sci. NS-20, 825 (1973);

C. Pellegrini and M. Sand, PEP Note 258 (1977).

2 . See for example, E.D. Courant and A.M. Sessler, Rev. Sci. Instruments 37, 1579 (1966).

3. See for example, B.V. Chirikov, Physics Report Vol. 52, No. 5 (1979). 